\title{
Ethics and amphibians
}

Robert M. May

\section{A statistical study shows convincingly that a technique for marking frogs in ecological field experiments compromises the results. Present practices need a rethink - and not only for practical reasons.}

lis t was 25 years ago, at an ecology seminar at Princeton University, that I first learned of the standard method for 'marking' individual newts or other amphibians (Fig. 1) by clipping their toes. In this way, each individual can be identified by the unique combination of digits removed. I remember being impressed by the elegance of the experiments concerned-but even more impressed by the casual barbarity of the toe clipping.

Seeking to avoid a larger ethical minefield, I asked whether such removal of digits would affect survival, particularly in more heavily clipped individuals, thus compromising the conclusions. My question was swept aside as silly (the sort of thing you might expect a theoretician to ask). But it now appears to have been answered. Writing in the Journal of Applied Ecology, McCarthy and Parris ${ }^{1}$ find that "toe clipping reduces the return rate [recapture of marked individuals] by $4-11 \%$ for each toe removed after the first, assuming the effect is the same for all toes".

The intervening years have, indeed, seen studies of the possible adverse effects of toe clipping of amphibians, including inflammation and infection of feet and limbs. Some of these studies indicate lower rates of return of marked individuals, others have found no such effects; Williamson and Bull $^{2}$ give a good review.

In an earlier paper, Parris and McCarthy ${ }^{3}$ suggested that these apparent inconsistencies arise from a lack of statistical power (sample sizes too small for statistically significant conclusions to be drawn) in some previous work, rather than from the absence of real effects. They estimated that return rates decline by $6-18 \%$ for each toe removed after the first. In this earlier paper ${ }^{3}$, however, they could not provide meaningful confidence intervals or a measure of how the effects would change with the number of toes removed.

The new paper $^{1}$ gives a Bayesian reanalysis of data drawn from four published studies on the return rate of toe-clipped frogs: 1,333 individuals of Crinia signifera (a small ground-dwelling Australian frog) with up to seven toes removed ${ }^{2} ; 306$ individuals of the same species with between two and four toes removed ${ }^{4} ; 33$ individuals of Bufo fowleri (a large ground-dwelling frog from eastern United States) with up to eight toes removed $^{5}$; and 1,307 individuals of Hyla labialis (a medium-sized tree frog from the

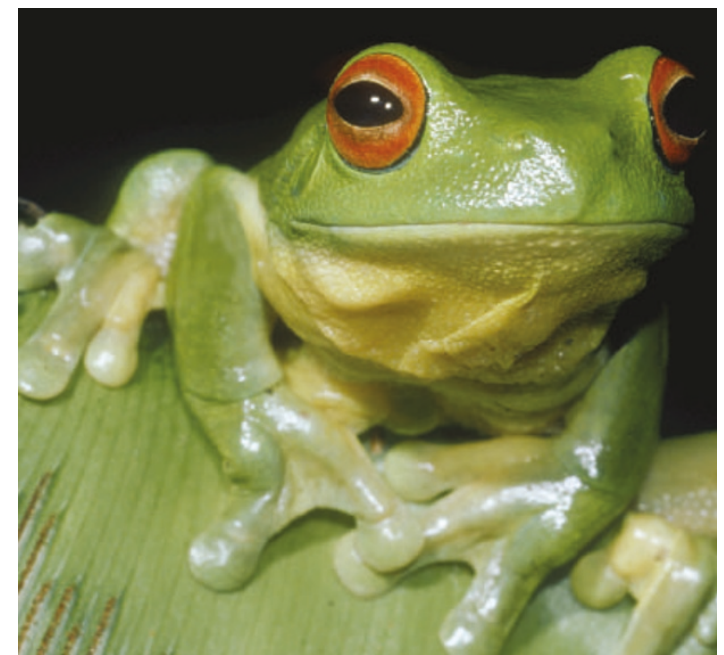

Figure 1 Toe show. Frogs are often the subject of studies involving toe clipping.

Colombian Andes) with up to seven toedisks removed ${ }^{6}$. The authors' Bayesian model, employing a freely available statistical program", helps "define the relationship between the number of frogs that are recaptured and the effect of toe-clipping as a function of the number of toes removed"1.

The estimated decline in return rate of $4-11 \%$ for each toe clipped assumed that the adverse effects are independent of the total number of toes clipped. McCarthy and Parris also provide a more refined analysis, allowing effects of toe removal to change linearly with the number removed. This indicated, for example, that the removal of a second toe reduced return rate by $3.5 \%$ (with a $95 \%$ "creditability interval" of $0-7 \%$ ), whereas return rate was reduced by $30 \%$ (95\% "creditability interval" of 20-39\%) on removal of an eighth toe. Overall, the cumulative effect of toe clipping appears to be such that the return rate for frogs with two toes removed was around $96 \%$ of those with one toe removed, declining to $28 \%$ for the return of frogs with eight toes gone.

The authors justifiably conclude that these effects should be more explicitly recognized in future studies that use toe clipping to mark individuals in ecological studies. They also tellingly add that "our study has important implications for the ethical treatment of animals, the continued use of clipping to mark species of conservation concern, and the removal of multiple toes from an individual frog or toad". It seems to me that all these conclusions apply to all amphibians.

More generally, I see McCarthy and Parris's paper as a notable addition to a growing literature that raises both practical and larger ethical questions about time-honoured procedures in some ecological field studies. There are obvious parallels with the recent study of long-term effects of flipper tags on penguins, by Gauthier-Clerc et al. ${ }^{8}$. This work attracted considerable media attention with its finding, after five years' work on king penguins implanted with electronic tags (some also with flipper bands and others not), that "banding results in later arrival at the colony for courtship in some years, lower breeding probability and lower chick production". Gauthier-Clerc et al. also found that unbanded king penguin chicks had roughly twice the survival rate after 2-3 years of those encumbered with flipper bands.

Scientific and medical understanding gained by the use of non-human animals in laboratory studies is widely recognized as producing great benefits, primarily for humans but also for other animals themselves. At the same time, such research is properly strictly regulated. And I for one welcome the attention increasingly given to the rights of non-human animals by philosophers such as Peter Singer. But field studies of the ecology and behaviour of non-human animals can also raise difficult questions of costs and benefits. As a sixth wave of mass extinction looms, conservation biologists desperately need the knowledge that comes from such field studies. As the work described here clearly shows, however, there are good reasons why we need to think more carefully about some present practices.

Robert M. May is in the Department of Zoology, University of Oxford, Oxford OX1 3PS, UK. e-mail: robert.may@zoology.oxford.ac.uk

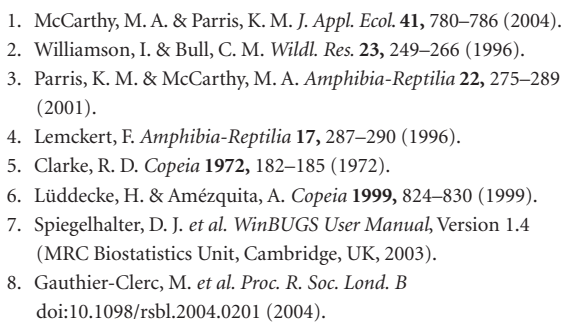

